\title{
Perineal Groove: Report of Three Cases
}

\section{Granillo Fernández S*, Garone A, Torres Molina L, Luty G, Bello C, Ripoli M, Rolotti M, Goitia J, Gomez Peral C and Rositto A}

Department of Dermatology, Hospital Sor Maria Ludovica, La Plata, Argentina

*Corresponding author: Granillo Fernández S, Department of Dermatology, Hospital Sor Maria Ludovica, La Plata, Argentina, Tel: (0221)482-1200; E-mail: sofiagranillo@hotmail.com

Received date: January 06, 2018; Accepted date: February 16, 2018; Published date: February 28, 2018

Copyright: ( 2018 Granillo SF, et al. This is an open-access article distributed under the terms of the Creative Commons Attribution License, which permits unrestricted use, distribution, and reproduction in any medium, provided the original author and source are credited.

\begin{abstract}
Perineal groove is an unknown congenital anomaly of the lower perineum. It has been described as a congenital reddish wet sulcus extending from the fourchette to the anterior anus. The three cases hereby presented were first misdiagnosed. The aim of this presentation is to make pediatricians and paediatric dermatologists aware of this condition in order to avoid unnecessary interventions.
\end{abstract}

Keywords: Perineal groove; Rare; Self-limiting

\section{Introduction}

Perineal groove was first reported in 1968 by Stephens as a congenital anomaly of the lower perineum. Its incidence seems to be underestimated. Twenty-three cases have been published among which only one male case has been reported [1].

It has been described as a congenital reddish wet sulcus extending from the fourchette to the anterior anus. It is generally asymptomatic and self-limiting.

\section{Case 1}

A female new-born was treated in Bolivia for diaper rash on several occasions. Eighteen months later, she moved to Argentina. She presented at the Emergency Department and was referred to the Dermatology Department for suspected sexual abuse.

On examination, the girl had a lineal reddish superficial lesion extending from the fourchette to the anus (Figure 1).

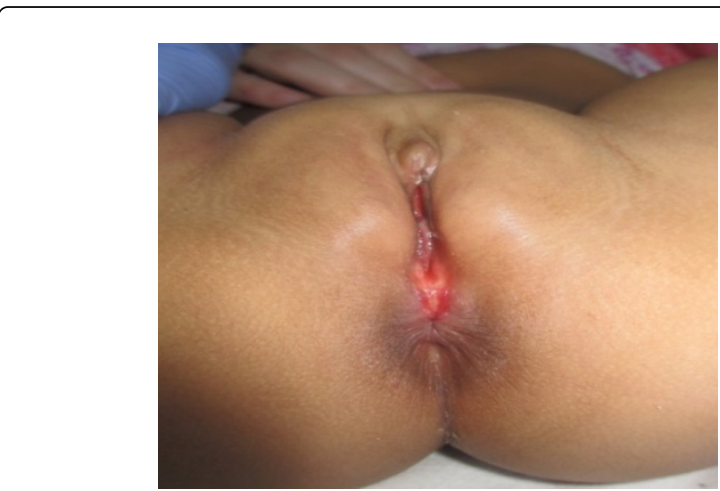

Figure 1: Erythematous wet groove between the vulva and the annuls.

On deeper examination, a whitish background is noticed (Figure 2). It was asymptomatic since birth.

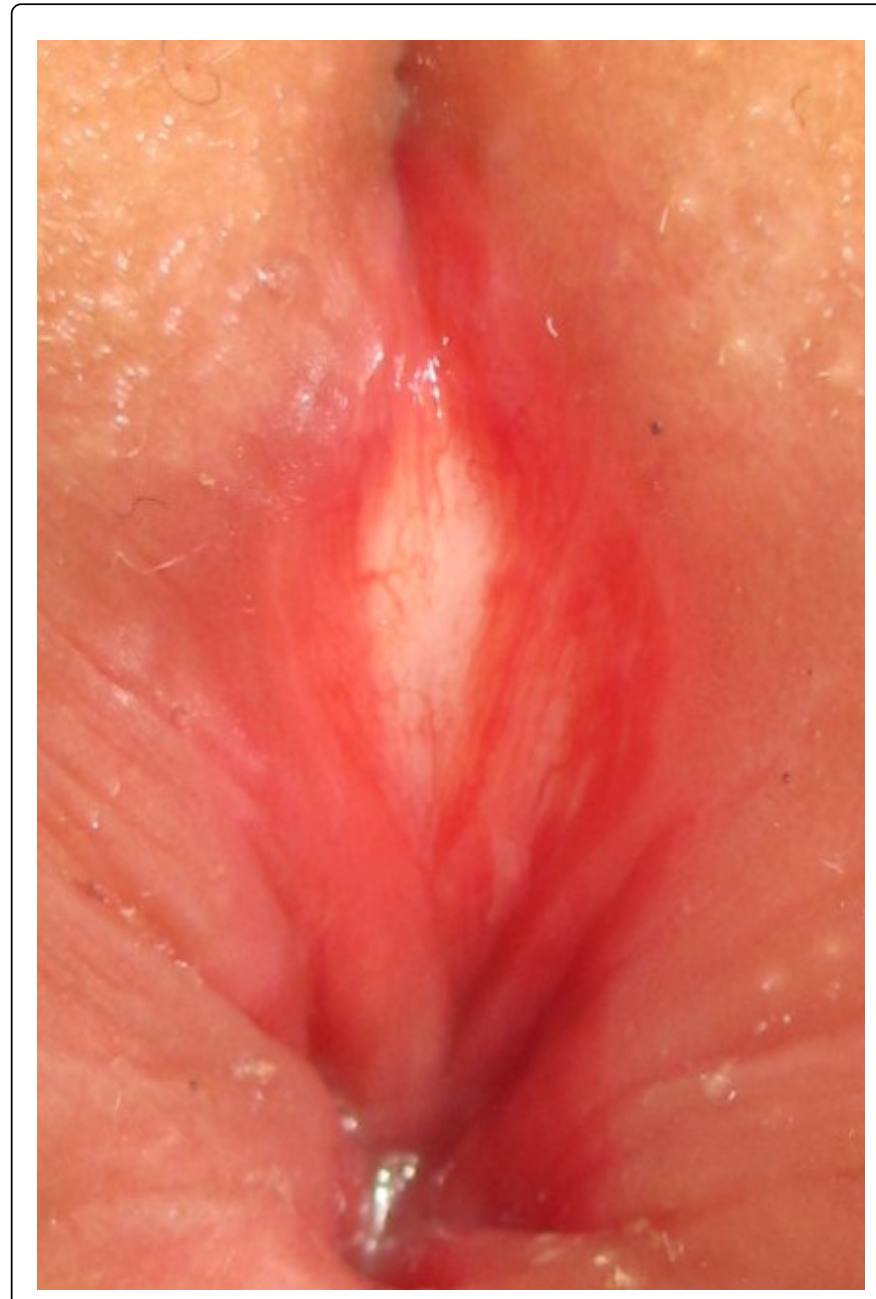

Figure 2: Groove with a whitish background.

As the lesion was congenital and there were no symptoms compatible with sexual abuse, the girl was diagnosed with perineal groove. 


\section{Case 2}

A 1-year-old girl had been treated with different topical agents for diaper rash without any improvement.

On physical examination, the lesion was almost imperceptible. It was slightly erythematous and extends from fourchette to the anus. It showed a mucus appearance with raised rims. Symptoms were not reported (Figure 3).

These findings supported the diagnosis of perineal groove.

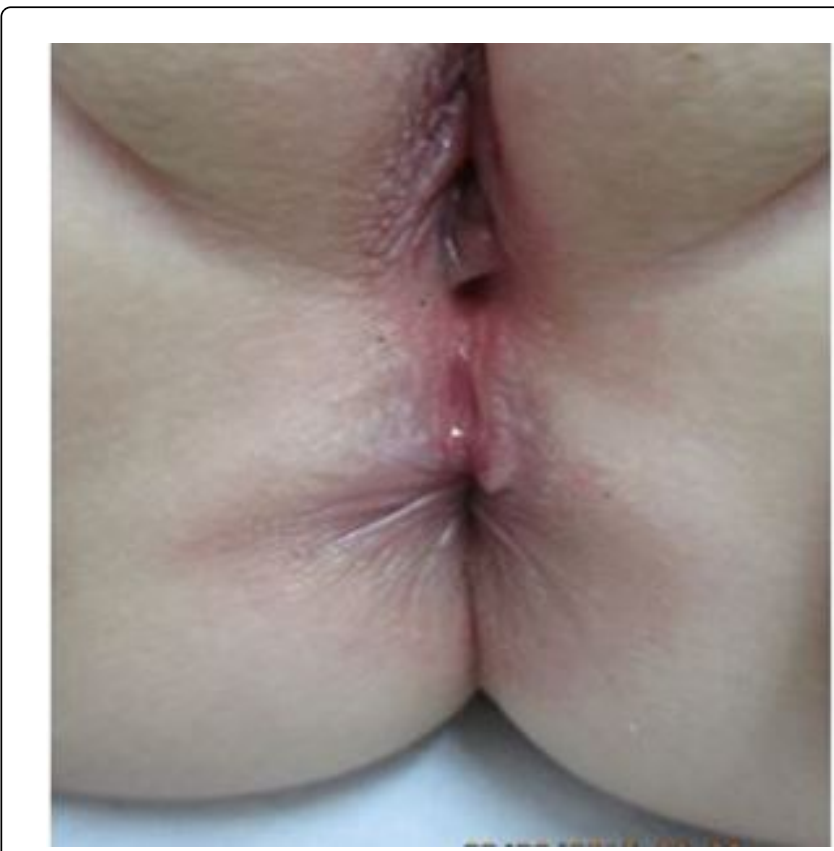

Figure 3: Linear lesion with a mucus appearance and raised rims.

\section{Case 3}

A 3-month-old female infant had been referred for a congenital lesion in the anterior anus with the diagnosis of diaper rash. She had received multiple treatments without improvement.

The lesion was not painful. Erosion and occasional bleeding were reported (Figure 4).

Given that she showed a congenital erythematous mucous linear lesion, the diagnosis of perineal groove was made.

In the three cases, abdominal ultrasound was performed to exclude associated malformations. Normal findings were found. The patients have been followed-up without the need of any treatment. So far, complications have not been reported.

\section{Discussion}

Perineal groove is a congenital anomaly of the lower perineum, whose incidence is unclear, although is believed to be higher since the condition might be underdiagnosed [1]. Twenty-three cases have been published among which only one male case has been reported [1,2].

The etiopathology remains unclear. It is thought that it is produced by an embryonic defect occurring in the 6-8th developmental weeks. It has been suggested that perineal groove could be due to a failure in the fusion of medial genital folds altering the formation of the perineum in the midline. It has also been speculated that it may be caused either by a relic of the open cloacal duct or an urorectal septum developmental defect [3].

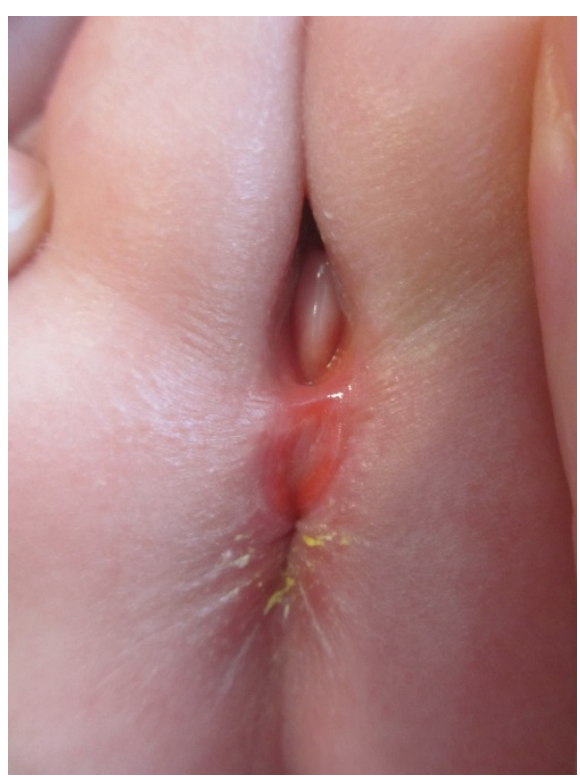

Figure 4: Well-demarcated erythematous superficial erosion anterior to the anus.

Although perineal groove has been described as a congenital reddish wet sulcus extending from the fourchette to the anterior anus, it is asymptomatic in most of the cases [2-4]. It is considered to be a self-limiting condition but it may take two years to become epithelialized [1,2]. In rare cases, there is an association with other urological or genital anomalies such as perineal ectopic anus, hypospadias, imperforated anus, rectal prolapse or urinary tract malformation $[2,5]$.

It is clinically diagnosed without biopsy. Histological evaluation of the resected area varied from a non-keratinized stratified squamous epithelium without sebaceous glands or sweat glands or hair follicles to a simple columnar or cuboidal epithelium of a rectal type mucosa with intervening area of a non-keratinized stratified squamous epithelium. These findings resembled anorectal transitional zone epithelium, which implied that this malformation is probably associated with an embryologic defect during urorectal septum development [3,5].

Differential diagnosis includes sexual abuse, erosive diaper rash, infections ulcers, Lipschutz ulcers, infantile haemangioma, Behcet's disease and liquen esclerosus. In our cases, sexual abuse was excluded due to the absence of other signs such us anal fissure, rectal tears or vaginal discharge. Likewise, diaper rash was also excluded because the lesions were described as a congenital lineal wet sulcus.

Perineal groove is generally asymptomatic, except when complications occur, such as constipation in which muscular fibers are abnormally distributed leading to sphincter dysfunction. Urinary and skin infections can also be reported [7]. 
Patients should be followed up until the lesion is completely reepithelialized. Surgery is recommended when the lesion is not epithelialized by the age of two, complications occur or there is aesthetic disfigurement $[2,6,7]$.

\section{Conclusions}

Perineal groove is a rare condition that is generally unknown by paediatricians and dermatologists. It should be included in the differential diagnosis of lesions involving the diaper area. Knowing this entity could help prevent unnecessary interventions and/or surgeries.

\section{References}

1. Sekaran P, Shawis R (2009) Perineal groove: a rare congenital abnormality of failure of fusion of the perineal raphe and discussion of its embryological origin. Clin Anat 22: 823-825.
2. Diaz L, LevyML, Kalajian A, Metry D (2014) Perineal groove: a report of 2 cases. JAMA Dermatol 150: 101-102.

3. Stephens FD (1968) The female anus, perineum and vestibule. Embryogenesis and deformities. Aust N Z J Obstet Gynaecol 8: 55-73.

4. Mullassery D, Turnock R, Kokai G (2016) Perineal groove. J Pediatr Surg 41: e41-e43.

5. Harsono M, Pourcyrous M (2016) Perineal Groove: A Rare Congenital Midline Defect of Perineum. Am J Perinatol Rep 6: e30-e32.

6. Esposito C, Giurin I, Savanelli A, Alicchio F, Settimi A (2011) Current trends in the management of pediatric patients with perineal groove. J Pediatr Adolesc Gynecol 24: 263-265.

7. Chatterjee SK, Chatterjee US, Chatterjee U (2003) Perineal groove with penoscrotal hypospadias. Pediatr Surg Int 19: 554-556. 